\title{
Hexane extracts of Polygonum multiflorum improve tissue and functional outcome following focal cerebral ischemia in mice
}

\author{
SOO VIN LEE ${ }^{1}$, KYUNG HA CHOI ${ }^{1}$, YOUNG WHAN CHOI ${ }^{2}$, JIN WOO HONG ${ }^{1}$, \\ JIN UNG BAEK ${ }^{1}$, BYUNG TAE CHOI ${ }^{1}$ and HWA KYOUNG SHIN ${ }^{1}$ \\ ${ }^{1}$ School of Korean Medicine, Pusan National University, Yangsan, Gyeongnam 626-870; ${ }^{2}$ College of Natural \\ Resources and Life Sciences, Pusan National University, Gyeongnam 627-706, Republic of Korea
}

Received July 22, 2013; Accepted February 5, 2014

DOI: $10.3892 / \mathrm{mmr} .2014 .1943$

\begin{abstract}
Polygonum multiflorum is a traditional Korean medicine that has been utilized widely in East Asian countries as a longevity agent. Clinical studies have demonstrated that Polygonum multiflorum improves hypercholesterolemia, coronary heart disease, neurosis and other diseases commonly associated with aging. However, scientific evidence defining the protective effects and mechanisms of Polygonum multiflorum against ischemic stroke is incomplete. In the present study, we investigated the cerebrovascular protective effects of Polygonum multiflorum against ischemic brain injury using an in vivo photothrombotic mouse model. To examine the underlying mechanism of action, we utilized an in vitro human brain microvascular endothelial cell (HBMEC) culture system. Hexane extracts (HEPM), ethyl acetate extracts (EAEPM) and methanol extracts (MEPM) of Polygonum multiflorum $(100 \mathrm{mg} / \mathrm{kg})$ were administered intraperitoneally $30 \mathrm{~min}$ prior to ischemic insult. Focal cerebral ischemia was induced in $\mathrm{C} 57 \mathrm{BL} / 6 \mathrm{~J}$ mice and endothelial nitric oxide synthase knockout (eNOS KO) mice by photothrombotic cortical occlusion. We evaluated the infarct volume, as well as neurological and motor function, $24 \mathrm{~h}$ after ischemic brain injury. Following ischemic insult, HEPM induced a significant reduction in infarct volume and subsequent neurological deficits, compared with EAEPM and MEPM. HEPM significantly decreased infarct size and improved neurological and motor function, which was not observed in eNOS KO mice, suggesting that this cerebroprotective effect is primarily an eNOS-dependent mechanism. In vitro, HEPM effectively promoted NO production, however these effects were inhibited by the NOS inhibitor, L-NAME and the PI3K/Akt
\end{abstract}

Correspondence to: Professor Hwa Kyoung Shin, Division of Meridian and Structural Medicine, School of Korean Medicine, Pusan National University, 49 Busandae hak-ro Multgeum-eup, Yangsan, Gyeongnam 626-870, Republic of Korea

E-mail: julie@pusan.ac.kr

Key words: Polygonum multiflorum, endothelial nitric oxide synthase, focal cerebral ischemia inhibitor, LY-294002. Furthermore, HEPM treatment resulted in increased phosphorylation-dependent activation of Akt and eNOS in HBMEC, suggesting that HEPM increased NO production via phosphorylation-dependent activation of $\mathrm{Akt}$ and eNOS. In conclusion, HEPM prevents cerebral ischemic damage through an eNOS-dependent mechanism, and thus may have clinical applications as a protective agent against neurological injury in stroke.

\section{Introduction}

Stroke is considered to be one of the leading causes of adult disability and mortality worldwide (1). Ischemic stroke is caused by cerebral thrombosis or embolism, which decreases cerebral blood flow (CBF) and triggers a series of deleterious biochemical events, including oxidative stress, activation of inflammatory mediators, upregulation of proteases and modulation of endothelial nitric oxide (NO) synthase (eNOS) (2). Delivery of the NOS substrate L-arginine, pharmacological NO donors, NO gas or overexpression of NOS proteins appears to protect against ischemic stroke (3). Endothelial NO release is enhanced through the direct phosphorylation of eNOS by the protein kinase Akt downstream of PI3K (4), and activation of eNOS/Akt leads to increased CBF, decreased cerebral infarction size and improved neurological deficit following cerebral ischemia (5). Thus, chemical or biological molecules that regulate eNOS activity have the potential for use as therapeutic drugs in the treatment of ischemic stroke.

Polygonum multiflorum Thunb. (Polygonaceae) has been widely utilized as a longevity agent in East Asian countries. Several clinical studies have revealed that Polygonum multiflorum is able to improve hypercholesterolemia, coronary heart disease, neurosis and other diseases commonly associated with aging (6). Polygonum multiflorum and its extracts have been reported to exert various pharmacological effects, including anti-oxidation, anti-inflammation and lipid regulation, as well as improving learning and memory (7-10). Polygonum multiflorum has also been reported to exert neuroprotective effects. For example, stilbene glycoside (2,3,5,4'-tetrahydroxystilbene2-O- $\beta$-D-glucoside), a major bioactive compound of Polygonum multiflorum, has been identified as protective against ischemia/reperfusion injury and $\mathrm{MPP}^{+}$-induced neurotoxic damage $(11,12)$. The accumulative evidence suggests that 
this medicine may be a reliable agent in the development of prevention and treatment strategies for permanent ischemic brain injury. In this study, we used an in vivo ischemic model of cortical infarction and an in vitro human brain microvascular endothelial cell (HBMEC) culture system to investigate the cerebrovascular protective effects of hexane extracts of Polygonum multiflorum (HEPM) against ischemic brain injury, and to define the underlying mechanisms that explain these effects.

To accomplish this, we examined the effects of HEPM on cerebral infarct, neurological and motor function and eNOS signaling using a photothrombotic mouse model in C57BL/6J and eNOS knockout (KO) mice. The results suggest that HEPM exerts cerebrovascular protective action against acute cerebral ischemic damage through an eNOS-dependent mechanism and thus may serve as a novel pharmacological therapeutic in the treatment of ischemic stroke.

\section{Materials and methods}

Preparation of Polygonum multiflorum extract. Dried roots of Polygonum multiflorum were purchased from Hwa Lim Natural Drug Co., Ltd. (Busan, Korea) in September 2010 and authenticated by one of the authors (Jin Woo Hong). A voucher specimen (accession number, PDRLCW-1) was deposited in the Plant Drug Research Laboratory of Pusan National University (Miryang, Korea). The dried roots of Polygonum multiflorum $(1.0 \mathrm{~kg}$ ) were ground to a fine powder, after which they were subjected to successive extraction at room temperature with n-hexane, ethyl acetate and methanol. Briefly, filtration and evaporation of HEPM was performed under reduced pressure at $45^{\circ} \mathrm{C}$, followed by lyophilization, which yielded a white powder of hexane extracts $(2.59 \mathrm{~g})$. Sequential extraction of the remaining powder was performed using ethyl acetate and methanol to yield ethyl acetate extracts (EAEPM; $9.30 \mathrm{~g}$ ) and methanol extracts (MEPM; $150.40 \mathrm{~g}$ ) of Polygonum multiflorum, respectively. Finally, the solid form of extract was dissolved with dimethyl sulfoxide (DMSO) for use in subsequent experiments.

Focal cerebral ischemia. Male mice (C57BL/6J and eNOS deficient; weighing, 20-25 g) were housed under diurnal lighting conditions and allowed food and tap water ad libitum. All animal procedures were conducted in accordance with the institutional guidelines for animal research, and were approved by the Pusan National University - Institutional Animal Care and Use Committee (PNU-IACUC; Busan, Republic of Korea) on ethical procedures and scientific care (PNU-2011-000367). Anesthesia was achieved by face maskdelivered isoflurane (2\% induction and $1.5 \%$ maintenance, in $70 \% \mathrm{~N}_{2} \mathrm{O}$ and $30 \% \mathrm{O}_{2}$ ). Sufficient depth of anesthesia was confirmed by the absence of cardiovascular changes in response to a tail pinch. Rectal temperature was kept at $36.5-37.5^{\circ} \mathrm{C}$ using a Panlab thermostatically controlled heating mat (Harvard Apparatus, Holliston, MA, USA). To determine whether Polygonum multiflorum was able to protect against ischemic stroke, EAEPM, MEPM and HEPM (100 mg/kg, intraperitoneally) were separately administered to the mice $30 \mathrm{~min}$ prior to ischemic insult. Focal cerebral ischemia was then induced using the photothrombotic cortical ischemia model (13). Briefly, $0.1 \mathrm{ml}$ of a $10 \mathrm{mg} /$ ml solution of Rose bengal (Sigma-Aldrich, St. Louis, MO, USA) in sterile saline was injected intraperitoneally $5 \mathrm{~min}$ prior to illumination. The mice were then placed in a stereotaxic frame and the midline scalp was incised, pericranial tissues were dissected, and the bregma and lambda points were identified. A fiber optic bundle with a KL1500 LCD cold light source (Carl Zeiss, Jena, Germany) and a $4 \mathrm{~mm}$ aperture was then centered $2 \mathrm{~mm}$ laterally from the bregma using a micromanipulator. Following this, the brain was illuminated through the intact skull for $15 \mathrm{~min}$, the surgical wound was then sutured and the mice were allowed to recover from anesthesia. Brains were removed $24 \mathrm{~h}$ following ischemic insult. Cerebral infarct size was determined based on analysis of 2,3,5-triphenyltetrazolium chloride (TTC)stained, 2-mm-thick brain sections and infarction areas were quantified using the iSolution full image analysis software (Image \& Microscope Technology, Vancouver, Canada). To account for and eliminate the effects of swelling/edema, infarction volume was calculated using an indirect measurement by summing the volumes of each section according to the following formula: contralateral hemisphere $\left(\mathrm{mm}^{3}\right)$ undamaged ipsilateral hemisphere $\left(\mathrm{mm}^{3}\right)$.

Neurological score and wire-grip test. Neurological deficit was scored in each mouse $24 \mathrm{~h}$ after the ischemic insults in a blinded fashion according to a $0-4$ scoring system in which $0=$ no deficit; 1 = forelimb weakness and torso turning to the ipsilateral side when held by the tail; 2 = circling to the affected side; 3 = unable to bear weight on the affected side and $4=$ no spontaneous locomotor activity or barrel rolling (14).

Vestibulo-motor function was assessed using a wiregrip test $24 \mathrm{~h}$ after cerebral ischemia (15). Briefly, mice were placed on a metal wire $(45 \mathrm{~cm}$ long) suspended $45 \mathrm{~cm}$ above protective padding and allowed to traverse the wire for $60 \mathrm{sec}$. The latency for which the mice remained on the wire within a $60 \mathrm{sec}$ interval was measured, and the wire grip score was quantified using the following 5-point scale in which $0=$ unable to remain on the wire for $30 \mathrm{sec}$; 1 = failure to hold on to the wire with fore paws and hind paws together; 2 = holding on to the wire with the fore and hind paws but not the tail; 3 = holding on to the wire using the tail along with the fore and hind paws; 4 = moving along the wire on four paws plus tail and finally $5=$ also ambulating down one of the posts used to support the wire. Tests were administered in triplicate and the average value was calculated for each mouse on each test day.

Determination of NO production in HBMECs. A membrane-permeable fluorescent indicator for NO, 4-amino5-methylamino-2',7'-difluorofluorescein diacetate (DAF-FM DA; Molecular Probes, Eugene, OR, USA) was utilized to detect HEPM-induced changes in NO production. DAF-FM DA is converted via a NO-specific mechanism to an intensely fluorescent triazole derivative (16). HBMECs were obtained from the Applied Cell Biology Research Institute (Kirkland, WA, USA) and cultured in endothelial growth medium-2 (EGM-2) using a MV Bullet kit system (Cambrex, 
Walkersville, MD, USA). Experiments were performed after 4-6 cell passages. Following reaching sub-confluency, the cells were incubated in endothelial cell basal medium (EBM; Cambrex) with HEPM and L-arginine for $10 \mathrm{~min}$, after which $5 \mu \mathrm{mol} / 1$ DAF-FM DA was loaded into the cells. Following incubation at $37^{\circ} \mathrm{C}$ for $5 \mathrm{~min}$, the cells were mildly washed twice using phosphate-buffered saline (PBS) to eliminate any interference. The NOS inhibitor, NG-nitro-L-arginine methyl ester (L-NAME; $100 \mu \mathrm{mol} / \mathrm{l})$ and acetylcholine $(30 \mu \mathrm{mol} / \mathrm{l})$ were used to validate the measurements. Fluorescence was detected using an Axiovert 200 fluorescence microscope (Carl Zeiss, Oberkochen, Germany).

Western blotting. To further assess the impact of HEPM on NO signaling, the phosphorylation of Akt at Ser473, AMPK at Thr172 and eNOS at Ser1177 in HBMEC was assessed by western blotting. Following treatment, HBMECs were washed in cold PBS buffer and then homogenized in lysis buffer [50 mM Tris- $\mathrm{HCl}(\mathrm{pH} 8.0), 150 \mathrm{mM} \mathrm{NaCl}$, $1 \mathrm{mM}$ EDTA, $1 \mathrm{mM} \mathrm{Na}_{3} \mathrm{VO}_{4}, 5 \mathrm{mM} \mathrm{NaF}, 1 \mathrm{mM}$ PMSF, $1 \%$ Triton $\mathrm{X}-100,10 \%$ glucose]. Proteins were isolated according to standard techniques, separated by $10 \%$ sodium dodecyl sulfate-polyacrylamide gel electrophoresis, and transferred onto a nitrocellulose membrane (Amersham Biosciences, Piscataway, NJ, USA). Immunoblot analysis was performed with anti-eNOS and anti-phospho-eNOS (Ser1177) antibodies (BD Biosciences, San Jose, CA, USA), anti-Akt and anti-phospho-Akt (Ser473) antibodies (Cell Signaling Technology, Inc., Danvers, MA, USA) and antiAMPK $\alpha$ and anti-phospho-AMPK $\alpha$ (Thr172) antibodies (Cell Signaling Technology, Inc.) followed by incubation with a secondary antibody conjugated with horseradish peroxidase. The intensity of chemiluminescence was measured using an ImageQuant LAS 4000 apparatus (GE Healthcare Life Sciences, Uppsala, Sweden). The membrane was reprobed with anti- $\beta$-actin antibody (Sigma-Aldrich) as an internal control.

Chemicals. Acetylcholine chloride and L-NAME were purchased from Sigma-Aldrich. LY-294002 and Compound $\mathrm{C}$ were purchased from Calbiochem Inc. (San Diego, CA, USA). n-Hexane, ethyl acetate and methanol were purchased from Fisher Scientific (Pittsburgh, PA, USA). All other chemicals were reagent grade.

Data analyses. All data are expressed as the mean \pm SEM. Statistical comparisons were performed using paired or unpaired Student's t-tests, and one-way analysis of variance (ANOVA) or two-way ANOVA for repeated measures followed by Fisher's protected least significant difference test. A $\mathrm{P}<0.05$ was considered to indicate a statistically significant result.

\section{Results}

Protective effects of Polygonum multiflorum extracts against ischemic brain injury. We investigated the protective effects of EAEPM, MEPM and HEPM using a focal cerebral ischemic mouse model. EAEPM, MEPM and HEPM (100 mg/kg, intraperitoneally) were separately administered to the mice
A
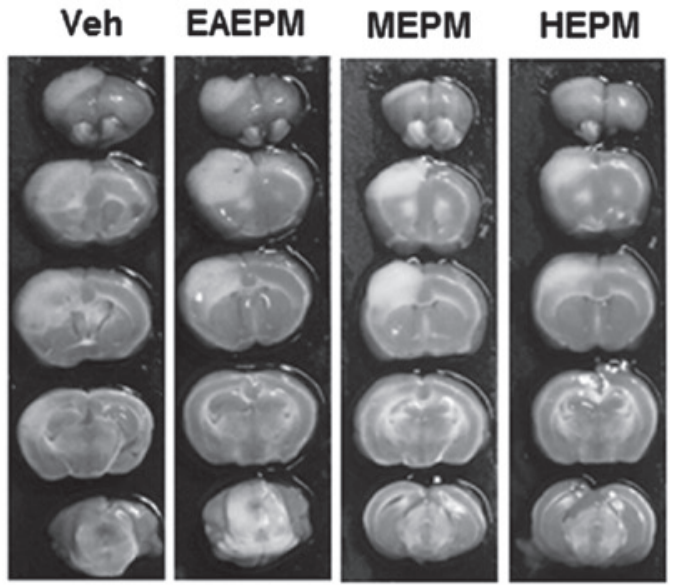

B
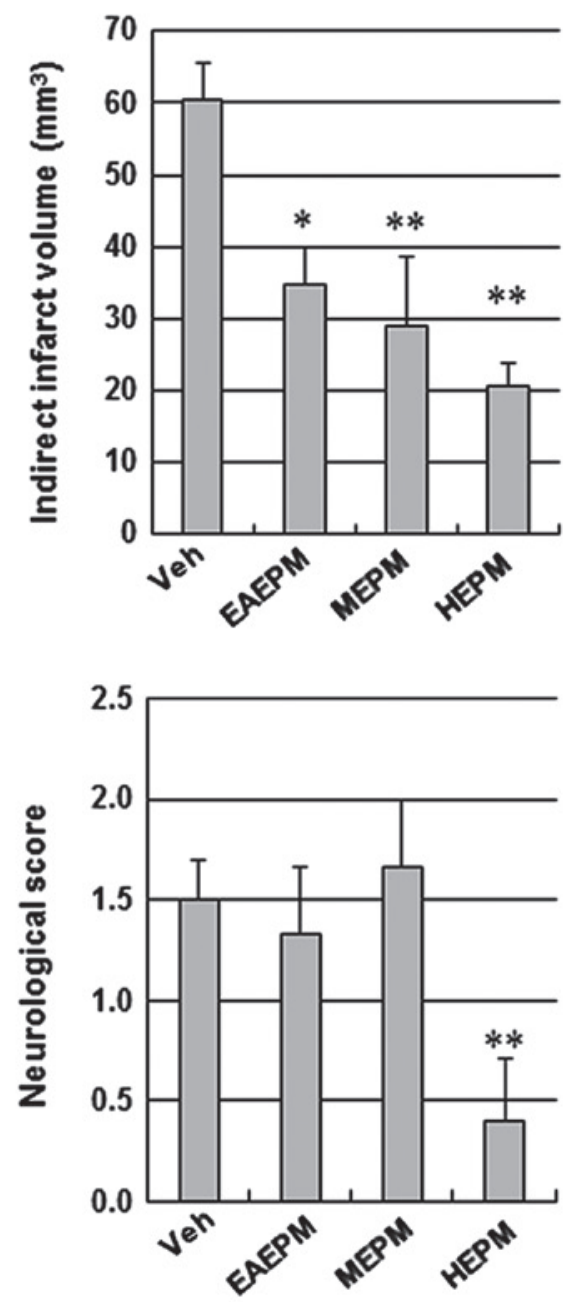

Figure 1. Effects of Polygonum multiflorum extracts on infarct volume and neurological function. (A) Representative images of coronal brain sections stained with TTC in Veh, EAEPM, MEPM and HEPM of Polygonum multiflorumtreated mice. Mice were intraperitoneally administered DMSO or $100 \mathrm{mg} / \mathrm{kg}$ EAEPM, MEPM and HEPM $30 \mathrm{~min}$ prior to ischemic insult. White indicates the infarct area. (B) Quantification of the infarct volume and neurological score $24 \mathrm{~h}$ following photothrombotic cortical ischemia. Data are expressed as the mean \pm SEM of five separate experiments. ${ }^{*} \mathrm{P}<0.05$ and ${ }^{* *} \mathrm{P}<0.01$ compared with the value in the vehicle group. TTC, 2,3,5-triphenyltetrazolium chloride; Veh, vehicle; EAEPM, ethyl acetate extracts of Polygonum multiflorum; MEPM, methanol extracts of Polygonum multiflorum; HEPM, hexane extracts of Polygonum multiflorum; DMSO, dimethyl sulfoxide. 

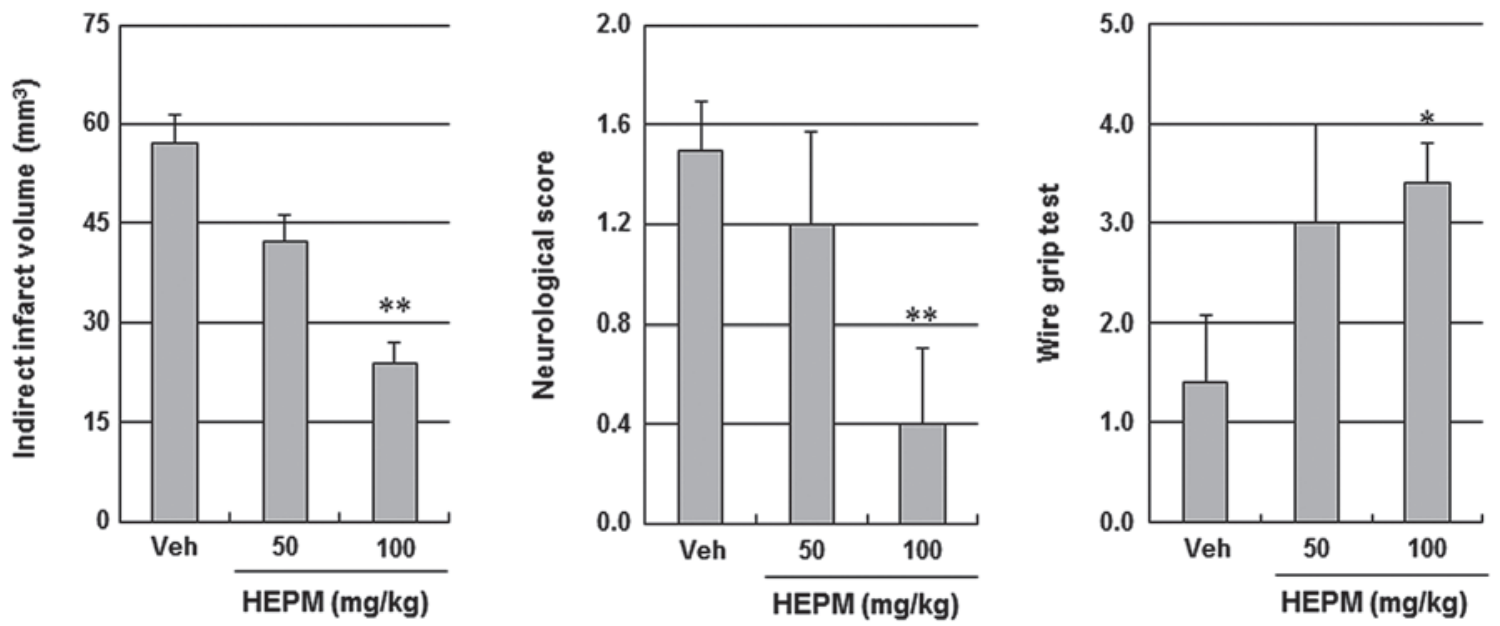

Figure 2. Concentration-dependent effects of HEPM on infarct volume and neurological and motor evaluation. Mice were intraperitoneally administered DMSO or 50 or $100 \mathrm{mg} / \mathrm{kg}$ HEPM 30 min prior to the ischemic insult. Quantification of the infarct volume, neurological and motor deficit were evaluated $24 \mathrm{~h}$ following photothrombotic cortical ischemia. Neurological function was assessed based on neurological score and vestibule-motor function by a wire grip test. Data are expressed as the mean \pm SEM of five separate experiments. ${ }^{*} \mathrm{P}<0.05$ and ${ }^{* *} \mathrm{P}<0.01$ compared with the value in the vehicle group. HEPM, hexane extracts of Polygonum multiflorum; DMSO, dimethyl sulfoxide.

A

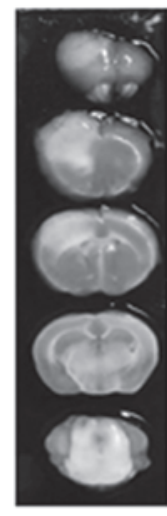

Veh

B

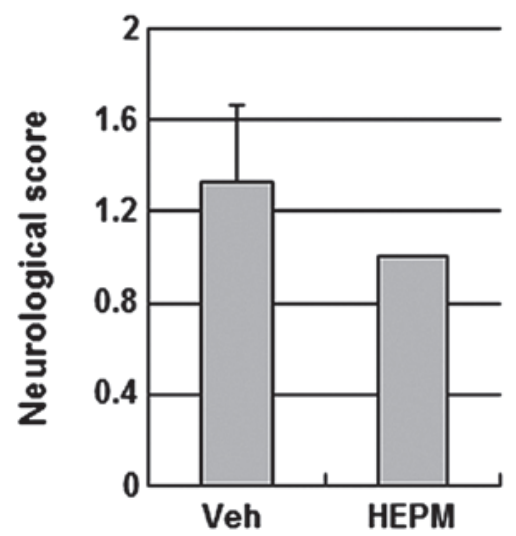

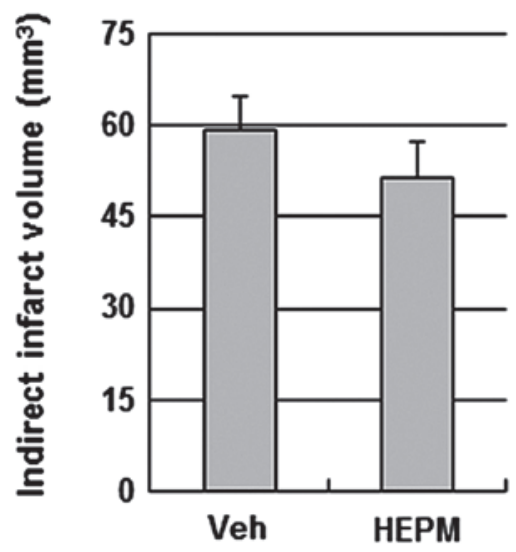

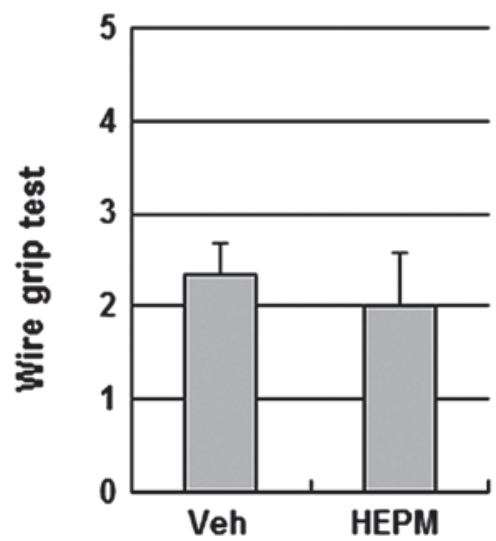

Figure 3. Effects of HEPM on the tissue and functional outcome in eNOS KO mice. (A) Representative images of the coronal brain sections stained with TTC in Veh and HEPM-treated eNOS KO mice. Mice were intraperitoneally administered DMSO or $100 \mathrm{mg} / \mathrm{kg} \mathrm{HEPM} 30$ min prior to the ischemic insult. White indicates the infarct area. (B) Neurological and motor deficit were assessed $24 \mathrm{~h}$ following brain ischemia. Data are expressed as the mean \pm SEM of four separate experiments. TTC, 2,3,5-triphenyltetrazolium chloride; HEPM, hexane extracts of Polygonum multiflorum; Veh, vehicle; eNOS KO, endothelial nitric oxide synthase knockout; DMSO, dimethyl sulfoxide.

30 min prior to ischemic insults. As summarized in Fig. 1, EAEPM, MEPM and HEPM all significantly decreased the cerebral infarct volume $\left(34.9 \pm 4.9 \mathrm{~mm}^{3}, 28.9 \pm 9.8 \mathrm{~mm}^{3}\right.$ and
$20.5 \pm 2.3 \mathrm{~mm}^{3}$, respectively) relative to the vehicle (Veh) treatment $\left(60.6 \pm 5.1 \mathrm{~mm}^{3}\right)$. Among these, HEPM exhibited the greatest protective effects against ischemic brain injury. 
A

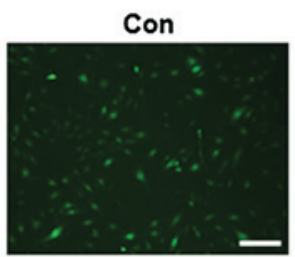

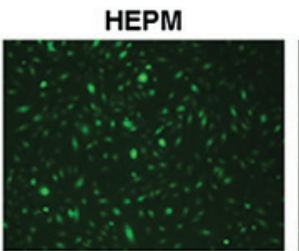
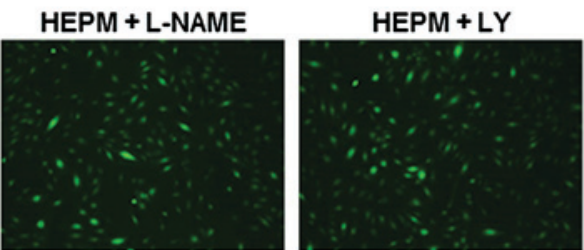

B

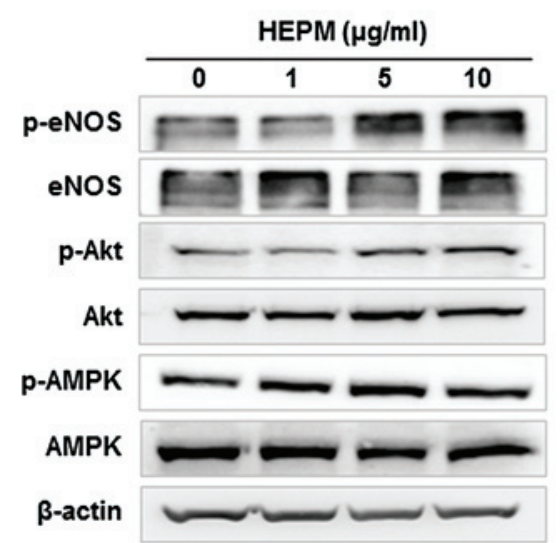

Figure 4. Effects of HEPM on NO production in HBMECs. (A) HBMECs were treated with HEPM (10 $\mu \mathrm{g} / \mathrm{ml})$ for $10 \mathrm{~min}$ following pretreatment with L-NAME (a NOS inhibitor, $100 \mu \mathrm{mol} / \mathrm{l}$ ), LY-294002 (LY, an inhibitor of PI3K/Akt, $10 \mu \mathrm{mol} / \mathrm{l}$ ) or Comp C, an AMPK inhibitor, $10 \mu \mathrm{mol} / \mathrm{l}$ ) for $30 \mathrm{~min}$. The intracellular levels of NO were then determined by fluorescence microscopy using DAF-FM DA. The scale bar represents $100 \mu \mathrm{m}$. (B) HBMECs were treated with the indicated concentrations of HEPM for $10 \mathrm{~min}$, after which the levels of phosphorylated proteins were determined by western blot analysis. HEPM, hexane extracts of Polygonum multiflorum; NO, nitric oxide; HBMECs, human brain microvascular endothelial cells; DAF-FM DA, 4-amino-5-methylamino-2',7'difluorofluorescein diacetate; Comp C, comound C; eNOS, endothelial nitric oxide synthase; p, phosphorylated.

Furthermore, only HEPM significantly improved neurological deficits. Therefore, HEPM was selected for further study.

Protective effects of HEPM against ischemic brain injury. When administered intraperitoneally at $50 \mathrm{mg} / \mathrm{kg}$ and $100 \mathrm{mg} / \mathrm{kg}$ $30 \mathrm{~min}$ prior to ischemic insult, HEPM decreased the infarct volume and improved the neurological and motor functions in a concentration-dependent manner (Fig. 2). To examine the contribution of eNOS signaling to the cerebroprotective action of HEPM, the impact of HEPM on ischemic brain injury was tested in eNOS KO mice. HEPM (100 mg/kg, intraperitoneally) did not reduce infarct volume or improve neurological and motor function in eNOS KO mice, suggesting that the cerebroprotective effects of HEPM are dependent on eNOS (Fig. 3).

Effects of HEPM on NO production in HBMECs. When HBMECs were incubated with $10 \mu \mathrm{g} / \mathrm{ml}$ HEPM, NO production was increased in vitro, as determined by the intensity of fluorescent DAF-FM (Fig. 4A). Previous studies have demonstrated that specific protein kinases, Akt and AMPK, are involved in eNOS phosphorylation and NO production $(4,17)$. To investigate the involvement of Akt and AMPK-dependent pathways in HEPM-induced NO production, we examined NO production in HBMECs co-treated with HEPM and L-NAME (a NOS inhibitor), LY-294002 (an inhibitor of PI3K/Akt) or Compound C (an AMPK inhibitor). HEPM-induced increases in NO production were effectively inhibited by L-NAME and LY-294002, but not by Compound C in HBMEC (Fig. 4A), indicating that HEPM-induced NO production was due to PI3K/Akt-dependent eNOS activation. Following this, we examined the effect of HEPM on the activation of these kinases and the phosphorylation of eNOS. HEPM treatment resulted in an increase in phosphorylation-dependent activation of Akt and eNOS in HBMEC (Fig. 4B). These results suggest that HEPM increased NO production via phosphorylationdependent activation of Akt and eNOS.

\section{Discussion}

The present study was conducted to examine the cerebrovascular protective effects of HEPM on ischemic brain injury in an in vivo photothrombotic mouse model, as well as to investigate its underlying mechanism in vitro in HBMECs. We identified that HEPM significantly decreased infarct volume and improved neurological and motor function. HEPM had no beneficial effect on tissue or functional outcome in eNOS KO mice, indicating that its cerebroprotective effects were mediated by eNOS. In vitro, HEPM promoted NO production, which was effectively inhibited by the NOS inhibitor, L-NAME and the PI3K/Akt inhibitor, LY-294002. Furthermore, HEPM treatment resulted in an increase in phosphorylation-dependent activation of Akt and eNOS in HBMECs, suggesting that HEPM increased NO production via phosphorylation-dependent activation of Akt and eNOS. These findings indicate that HEPM exerts cerebroprotective action through an eNOS-dependent mechanism. This study provides evidence that HEPM is a potential protective agent against ischemic brain injury.

Polygonum multiflorum is one of the most important traditional Korean medicines and is widely utilized in the treatment of diseases commonly associated with aging. In addition, the roots of the Polygonum multiflorum have been used to treat cardiovascular diseases, including atherosclerosis and hypertension (8) 
and have been reported to exert neuroprotective effects against ischemic brain injury. Specifically, long term pretreatment with the $50 \%$ ethanol extract of Polygonum multiflorum for 2 weeks significantly reduced cerebral ischemia-induced infarct volume in gerbils (18) and HEPM attenuated glutamate-induced neurotoxic damage in primary cultured cortical neurons (19). Furthermore, stilbene glycoside, a major bioactive compound of Polygonum multiflorum, exerts protective effects in an ischemic model of oxygen-glucose deprivation, followed by reperfusion and middle cerebral artery occlusion and $\mathrm{MPP}^{+}$-induced neurotoxic damage $(11,12)$. Therefore, this traditional medicine has attracted a great deal of attention and research interest in studies investigating the potential therapeutic application of extracts of Polygonum multiflorum against permanent ischemic brain injury. In the present study, we examined the protective effects of EAEPM, MEPM and HEPM on focal cerebral ischemia and revealed that HEPM most significantly reduced infarct volume and improved neurological function following photothrombotic cortical occlusion (Fig. 1). These findings suggest that of all the Polygonum multiforum extracts, HEPM has the most prominent neuroprotective effect against ischemic stroke.

Polygonum multiflorum and its extracts have been reported to exert various pharmacological effects, including antioxidation, anti-inflammation and lipid regulation $(7,8)$. Several mechanisms of action of Polygonum multiflorum on ischemic brain injury have been reported. For example, Polygonum multiflorum attenuated glutamate-induced neurotoxicity via the suppression of DR4 and the upregulation of Bcl-2, XIAP and cIAP-1, as well as via the inhibition of caspase activation, resulting in prevention of apoptosis of cortical neurons (19). Furthermore, one of the major active components extracted from Polygonum multiflorum, stilbene glycoside, has been shown to exert neuroprotective effects against ischemia/reperfusion brain injury in vitro and in vivo by reducing oxidative stress. Although there have been relatively extensive investigations of the antiapoptotic and anti-oxidative effects of Polygonum multiflorum in ischemic brain injury, no studies have described the eNOSdependent cerebrovascular protective effect of Polygonum multiflorum against ischemic stroke.

NO generated by eNOS in endothelial cells is important in vasorelaxation, inhibition of platelet aggregation, endothelial cell survival and angiogenesis, resulting in protection of the vasculature against various pathological conditions (20). For example, eNOS is an important mediator of CBF that is critical to the regulation of vascular tone and the maintenance of vascular integrity in cerebral vasculature (21). Accordingly, conditions that enhance eNOS protein expression and enzymatic activation may have beneficial effects on cerebrovascular disease through NO production (3). It has also been revealed that Polygonum multiflorum may have vasorelaxant abilities (22). Furthermore, stilbene glycoside enhanced NO and cGMP formation through the upregulation of endothelial NO synthase expression in vascular smooth muscle cells (23). Another study demonstrated in vivo that stilbene glycoside attenuated intimal hyperplasia and improved endothelial function in atherosclerotic rats, which was correlated with increased NO levels in the serum and aorta (24). Taken together, these findings suggest that Polygonum multiflorum is capable of inducing cerebrovascular protection through the elevation of NO production in ischemic stroke. However, this hypothesis had not been investigated until now. In the present study, the cerebroprotective action of HEPM was eliminated in the eNOS KO mice. Therefore, the beneficial effects of HEPM on ischemic injury are due, at least in part, to its vascular protective actions, which involve eNOSdependent mechanisms. Consistent with these findings, we identified that HEPM increased NO production in HBMECs, which was effectively inhibited by L-NAME, suggesting that HEPM induces eNOS-dependent NO production.

Following this, we examined the underlying mechanism of HEPM-induced NO production. The specific protein kinases, Akt and AMPK, have been demonstrated to be involved in eNOS phosphorylation and NO production $(4,17)$. We identified that HEPM-induced NO production was inhibited by the NOS inhibitor, L-NAME, as well as the PI3K/Akt inhibitor, LY-294002, but not by an AMPK inhibitor, Compound C, in HBMECs (Fig. 4A). In addition, HEPM treatment resulted in an increase in the phosphorylation-dependent activation of Akt and eNOS in HBMECs (Fig. 4B). Endothelial-derived $\mathrm{NO}$ production is not only associated with post-translational modulation of eNOS activity (25), but also with an increase in transcriptional expression (26). We demonstrated that HEPM increased endothelial NO production without altering the levels of eNOS and Akt protein expression as determined by western blotting. Therefore, our results suggests that HEPM increased endothelial NO synthesis by regulating eNOS activity, without changing its expression. Taken together, our findings demonstrate that HEPM promotes NO production through PI3K/Akt-dependent eNOS activation.

In conclusion, HEPM improves tissue and functional outcome in focal cerebral ischemic damage, while HEPMmediated cerebroprotective effects are absent in eNOS $\mathrm{KO}$ mice. HEPM also induces NO production through PI3K/Akt-dependent eNOS activation in HBMECs, indicating the obligatory role of endothelium-derived NO in mediating this effect. These results, coupled with data gathered from previous studies, have revealed the beneficial actions of Polygonum multiflorum on neuron and cerebral vasculature, and strongly suggest that this agent has potential applications as a novel therapeutic strategy in the prevention and treatment of ischemic stroke.

\section{Acknowledgements}

This study was supported by the R\&D program of MKE/KIAT (Establishment of Infra Structure for Anti-Aging Industry Support).

\section{References}

1. Roger VL, Go AS, Lloyd-Jones DM, et al: Heart disease and stroke statistics - 2011 update: a report from the American Heart Association. Circulation 123: e18-e209, 2011.

2. Allen CL and Bayraktutan U: Risk factors for ischaemic stroke. Int J Stroke 3: 105-116, 2008.

3. Endres M, Laufs U, Liao JK and Moskowitz MA: Targeting eNOS for stroke protection. Trends Neurosci 27: 283-289, 2004.

4. Dimmeler S, Fleming I, Fisslthaler B, Hermann C, Busse R and Zeiher AM: Activation of nitric oxide synthase in endothelial cells by Akt-dependent phosphorylation. Nature 399: 601-605, 1999.

5. Limbourg FP, Huang Z, Plumier JC, et al: Rapid nontranscriptional activation of endothelial nitric oxide synthase mediates increased cerebral blood flow and stroke protection by corticosteroids. J Clin Invest 110: 1729-1738, 2002. 
6. Xiao PG, Xing ST and Wang LW: Immunological aspects of Chinese medicinal plants as antiageing drugs. J Ethnopharmacol 38 167-175, 1993.

7. Liu Z, Liu Y, Chao Z, Song Z, Wang C and Lu A: In vitro antioxidant activities of maillard reaction products produced in the steaming process of Polygonum multiflorum root. Nat Prod Commun 6: 55-58, 2011.

8. Yang PY, Almofti MR, Lu L, et al: Reduction of atherosclerosis in cholesterol-fed rabbits and decrease of expressions of intracellular adhesion molecule-1 and vascular endothelial growth factor in foam cells by a water-soluble fraction of Polygonum multiflorum. J Pharmacol Sci 99: 294-300, 2005.

9. Um MY, Choi WH, Aan JY, Kim SR and Ha TY: Protective effect of Polygonum multiflorum Thunb on amyloid beta-peptide 25-35 induced cognitive deficits in mice. J Ethnopharmacol 104: 144-148, 2006.

10. Chan YC, Wang MF and Chang HC: Polygonum multiflorum extracts improve cognitive performance in senescence accelerated mice. Am J Chin Med 31: 171-179, 2003.

11. Wang T, Gu J, Wu PF, et al: Protection by tetrahydroxystilbene glucoside against cerebral ischemia: involvement of JNK, SIRT1, and NF-kappaB pathways and inhibition of intracellular ROS/RNS generation. Free Radic Biol Med 47: 229-240, 2009.

12. Sun FL, Zhang L, Zhang RY and Li L: Tetrahydroxystilbene glucoside protects human neuroblastoma $\mathrm{SH}-\mathrm{SY} 5 \mathrm{Y}$ cells against

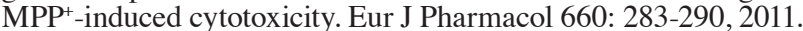

13. Lee JK, Park MS, Kim YS, et al: Photochemically induced cerebral ischemia in a mouse model. Surg Neurol 67: 620-625, 2007.

14. Li X, Blizzard KK, Zeng Z, DeVries AC, Hurn PD and McCullough LD: Chronic behavioral testing after focal ischemia in the mouse: functional recovery and the effects of gender. Exp Neurol 187: 94-104, 2004

15. Chen T, Liu W, Chao X, et al: Salvianolic acid B attenuates brain damage and inflammation after traumatic brain injury in mice. Brain Res Bull 84: 163-168, 2011.
16. Kojima H, Sakurai K, Kikuchi K, et al: Development of a fluorescent indicator for nitric oxide based on the fluorescein chromophore. Chem Pharm Bull (Tokyo) 46: 373-375, 1998.

17. Fisslthaler B and Fleming I: Activation and signaling by the AMP-activated protein kinase in endothelial cells. Circ Res 105 114-127, 2009.

18. Chan YC, Wang MF, Chen YC, Yang DY, Lee MS and Cheng FC: Long-term administration of Polygonum multiflorum Thunb. reduces cerebral ischemia-induced infarct volume in gerbils. Am J Chin Med 31: 71-77, 2003

19. Jang JY, Kim HN, Kim YR, et al: Hexane extract from Polygonum multiflorum attenuates glutamate-induced apoptosis in primary cultured cortical neurons. J Ethnopharmacol 145: 261-268, 2013.

20. Dudzinski DM and Michel T: Life history of eNOS: partners and pathways. Cardiovasc Res 75: 247-260, 2007.

21. Huang Z, Huang PL, Ma J, et al: Enlarged infarcts in endothelia nitric oxide synthase knockout mice are attenuated by nitro-Larginine. J Cereb Blood Flow Metab 16: 981-987, 1996.

22. Huang HC, Chu SH and Chao PD: Vasorelaxants from Chinese herbs, emodin and scoparone, possess immunosuppressive properties. Eur J Pharmacol 198: 211-213, 1991.

23. Xu XL, Huang YJ, Chen XF, Lin DY and Zhang W: 2,3,4',5-tetrahydroxystilbene-2-O- $\beta$-D-glucoside inhibits proliferation of vascular smooth muscle cells: involvement of NO/cGMP/PKG pathway. Phytother Res 26: 1068-1074, 2012.

24. Zhang W, Xu XL, Wang YQ, Wang CH and Zhu WZ: Effects of 2,3,4',5-tetrahydroxystilbene 2-O-beta-D-glucoside on vascular endothelial dysfunction in atherogenic-diet rats. Planta Med 75: $1209-1214,2009$

25. Fulton D, Gratton JP and Sessa WC: Post-translational control of endothelial nitric oxide synthase: why isn't calcium/calmodulin enough? J Pharmacol Exp Ther 299: 818-824, 2001.

26. MacRitchie AN, Jun SS, Chen Z, et al: Estrogen upregulates endothelial nitric oxide synthase gene expression in fetal pulmonary artery endothelium. Circ Res 81: 355-362, 1997. 